\title{
Finding out what's happened: Two procedures for opening emergency calls
}

Jakob Cromdal, Håkan Landqvist, Daniel Persson Thunqvist and Karin Osvaldsson

\section{Linköping University Post Print}

N.B.: When citing this work, cite the original article.

Original Publication:

Jakob Cromdal, Håkan Landqvist, Daniel Persson Thunqvist and Karin Osvaldsson, Finding out what's happened: Two procedures for opening emergency calls, 2012, Discourse Studies, (14), 4, 371-397.

http://dx.doi.org/10.1177/1461445612439960

Copyright: SAGE Publications (UK and US)

http://www.uk.sagepub.com/home.nav

Postprint available at: Linköping University Electronic Press

http://urn.kb.se/resolve?urn=urn:nbn:se:liu:diva-80622 
To appear in Discourse Studies (2012)

\title{
Finding out what's happened: \\ Two procedures for opening emergency calls
}

\author{
Jakob Cromdal $^{\mathrm{i}}$ \\ Department of Social and Welfare Studies \\ Linköping University \\ 60174 NORRKÖPING, SWEDEN \\ jakob.cromdal@liu.se
}

Håkan Landqvist

School of Education, Culture and Communication

Mälardalen University, Sweden

Daniel Persson-Thunqvist

Department of Behavioural Sciences and Learning

Linköping University, Sweden

Karin Osvaldsson

Department of Child Studies

Linköping University, Sweden

Running head: Two procedures for opening emergency calls

Word count: 8760 (body, transcripts included)

ABSTRACT: This article examines two corpora of telephone calls to the Swedish emergency services SOS-

Alarm. The focus of analysis is on the procedural consequentiality of the routine opening by the operator. In the first corpus, the summons are answered by identification of the service via the emergency number. In the second corpus, the protocol has been altered, such that the opening entails the emergency number combined with a standard query concerning the nature of the incident. Through sequential and categorial analysis of the two collections, we highlight the distinct trajectories of action ensuing from the two opening protocols. The standalone emergency number opening typically results in callers asking for a specific service. In contrast, opening turns which end with a direct query about the incident tend to solicit brief descriptions of the trouble. We discuss the benefits of the latter procedure in terms of topical progression and institutional relevance, proposing that the work of emergency assistance agencies worldwide might consider implementing opening routines with a similar design.

Key words: Conversation analysis; Emergency calls; Ethnomethodology; Helplines; Institutional interaction; Service calls; Studies of work; Talk-in-interaction. 
Jakob Cromdal is professor in educational practice at the Department of Social and Welfare Studies, Linköping University, Sweden. His research focuses on talk and social interaction among children and youth in a variety of mundane and institutional settings, including playgrounds, classrooms, detention homes and calls to the emergency services.

Håkan Landqvist works as a senior lecturer at Mälardalen University, Sweden. His primary research interest is in institutional discourse, and he has for instance analysed telephone calls to the Swedish Poisons Information Centre. A broader scope of research interests includes sociolinguistics as well as interactional linguistics.

Daniel Persson-Thunqvist holds a degree in Communication Studies (Linköping, 2003). He is a senior lecturer in sociology. His research interests are in the field of discourse studies, particularly in professional-lay interaction and workplace interaction.

Karin Osvaldsson received her PhD in Child studies at Linköping University in 2002. She is currently engaged in research on social interaction in various settings, including detention homes for troubled youth, emergency rescue calls and young persons' participation in internet notice boards and chat-rooms. 


\section{Finding out what's happened: Two procedures for opening emergency calls}

\section{Background}

In 1996, the number to the emergency rescue services in Sweden was officially changed from 90000 to 112 . This was a step towards the implementation of a European Union standard number for contacting local emergency rescue agencies (91/396/EEC). As part of the transition, the Swedish emergency rescue services SOS Alarm also modified the operator protocol for interrogating callers about the nature of their emergency and organising response activities. One immediately observable change concerned the standardised opening phrase through which emergency operators respond to the summons:

opening line prior to 1996 :

"Nittio Tusen" alternatively "SOS Nittio Tusen"

"Ninety Thousand" alternatively "SOS Ninety Thousand"

after 1996:

"SOS Ett Ett Två, vad har inträffat?"

"SOS One One Two, what has occurred?"

The dedicated emergency number is stated in both versions of the opening phrase. The explicit identification of the emergency resue services via the abbreviation "SOS", optional in the earlier routine, became a mandatory first-position element in the post 1996 opening 
phrase. But perhaps the most striking difference between the two routines is the concluding query of the latter, concerning what has "occurred" ii . In this article, we examine two collections of emergency call openings in which each of the two phrases is used by operators to respond to the summons. The focus is on the procedural consequentiality (Sacks, et al., 1974) of the two types of opening turns, particularly on their bearing on the callers' formulation of their reason for calling the emergency services. Our overall concern then, is with the ways through which conversational practice is organised to allow for the work of emergency call-taking and response management to take place (cf. Watson \& Sharrock, 1990). This approach turns on the assumption that public institutions can be analysed as ways of talking (e.g., Bittner, 1974; Garfinkel, 1969; Watson, 1986). Through such verbal conduct, participants can be seen to act on, and display for one another, any personal and organisational concerns relevant for the business at hand.

\section{Emergency call openings}

Previous work in conversation analysis has shown that openings in telephone calls are organised to allow the parties to establish an early sense of what type of call they are dealing with (e.g., Hopper, 1992; Schegloff, 1968; 1979a; 2002). Although such intersubjectivity arrangements are crucial to all social interaction, they are, perhaps, particularly explicit in service-related telecommunications. Here, the call taker's answer to the summons typically exhibits some distinctive features of the institution, including identification of the business, more or less explicit information concerning the professional services offered and other organisational relevances (e.g., Baker, et al., 2005; Danby, et al., 2005; Whalen \& Zimmerman, 1987; Watson, 1986). Such opening arrangements render the exchange its recognisably institutional character ${ }^{\mathrm{iii}}$.

A subset of service-related telephone calls which has received a fair deal of scholarly attention comprise citizens' calls for emergency assistance (see Cromdal, in press, for a review), and several studies have examined the opening exchanges between caller and call taker. Characteristic of such openings is the reduction (Whalen \& Zimmerman, 1987) achieved through methodic omission of such mundane routines as greeting exchanges and other common courtesies, and specialisation (Wakin \& Zimmerman, 1999), achieved via a standardised identification of the service, typically located to the second slot in the summons- 
answer (SA) sequence (Schegloff, 1968) thereby constituting the first spoken turn of the call. This design of the opening procedures is organisationally motivated. As Zimmerman (1992a) points out:

The trajectory of the call is thus anticipated in its opening, which projects a framework - the shell of a particular kind of activity - within which structurally and substantively relevant understandings of subsequent utterances can be achieved. The opening turns of the call, and in particular the components of the first turns of answerer and caller, regularly establish an identity set implicative for the nature of the business to follow. (p. 49-50)

Hence, a specialised context is produced in the opening of the call; a context that provides for the callers' recognition of certain commonsense understandings of the business that is routinely pursued within such settings. While the identification is invariably positioned in immediate response to the summons, there is some variation to how such turns are constructed. For instance, call takers may answer the summons by stating the dedicated emergency number, e.g., "Nine One One" (cf. Garcia \& Parmer, 1999), "1-1-8” (Paoletti, 2007), "Ninety Thousand" (Nordberg, 1999), thus establishing a "socially sanctioned inferential framework" (Wakin \& Zimmerman, 1999, p. 433) within which callers' ability to infer the correct identity and business of the service is presumed. Alternatively, identification may be achieved by explicit naming of the emergency service, e.g., "Emergency Centre" (Arminen, et al., 2008; Eglin, 1986), “Feuerwehr?"/"Fire Brigade?” (Bergmann, 1992), or "Police Desk" (Schegloff, 1968), occasionally including regional specification, such as "MidCity Emergency", (cf. Whalen \& Zimmerman, 1987) and "Newton Police" (Sharrock \& Watson, 1987). The two procedures may be combined, such that both the number and the identification of the service are given in the operator's answer to the summons, as in "Nine One One Emergency" (cf. Whalen \& Zimmerman, 1987), "SOS Ninety Thousand" (Nordberg, 1999). In addition, we find more complex opening phrases in the literature, such as "City West Nine One One, Agent Geltner" (Tracy \& Anderson, 1999), which combine regional information with the dedicated number as well as with personal and occupational identification of the call taker.

Such categorial identifications do not merely provide for the caller's recognition of the service. Rather, operators' responses to the summons partition the interlocutors into two 
mutually constitutive categories, or "standardised relational pairs" (Sacks, 1972), which we may call help-seeker and help-provider, where the mention (or other means of invokation) of one pair part is sufficient for the other to become relevant for the ongoing conduct $^{\mathrm{i}}$. Hence, the relevant "identity set" (Zimmerman 1992a) is established through ordinary practices of membership categorization: if the call taker answers the summons by categorising herself as a provider of emergency assistance, then that ascribes to the caller the corresponding identity as a seeker of such assistance. The local relevance of such a categorial identification is readily observable in instances when one of the parties treats their identity as problematic:

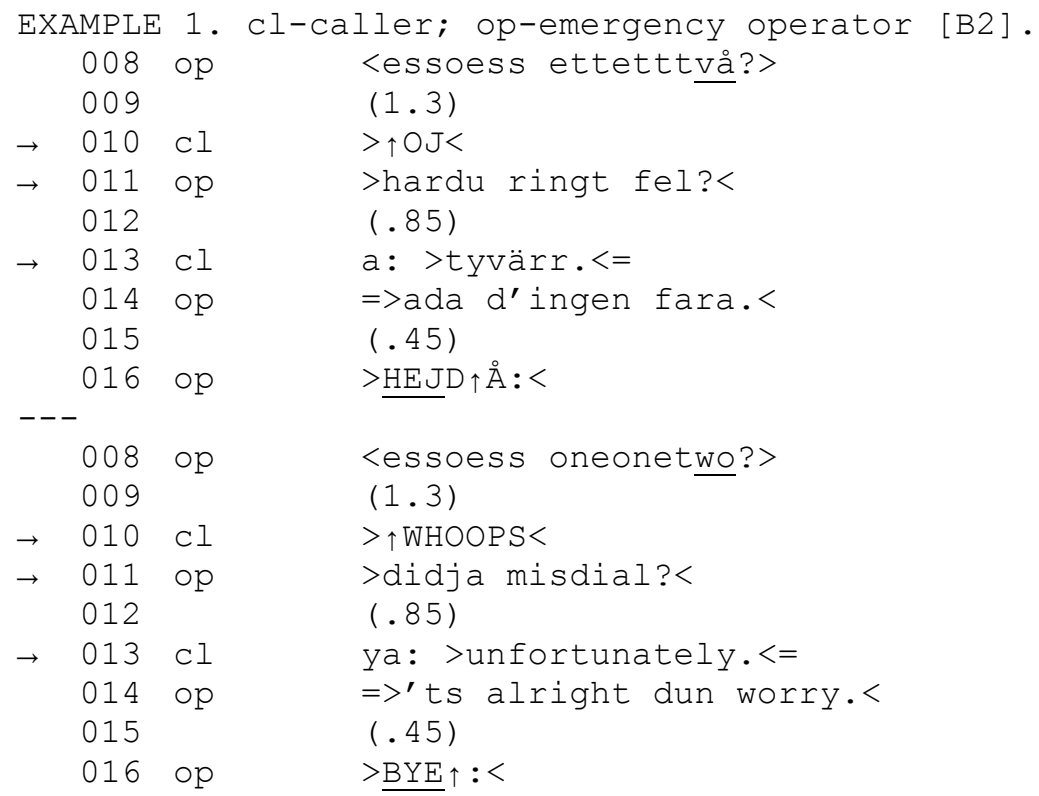

In response to the operator's identification, the caller produces a prosodically marked token of surprise "OJ" ("WHOOPS"). The operator then provisionally treats this as an indication of misdialling, which the caller confirms initiating an apology - acceptance sequence, before the operator moves on to terminate the call.

Clearly then, calling the emergency services is an accountable action. It is not to be done without good reason, and consequently the interaction in the very beginning of such calls is geared towards finding out that reason with as little delay as possible. In contrast to mundane telephone calls, where there may be little need to account for the call - or indeed, where there may be good grounds for minimising or countering the recongisability of the caller's reason for calling (cf. Sacks, 1992, Vol. 2, p. 165-171) - the organisation of openings in emergency calls locates an expectation on the caller to use the "first topic slot" (Whalen \& Zimmerman, 1987) to state or otherwise make inferrable a valid reason for the call. Again, the normative features of this organisation become observable when the expectation is not satisfied: 


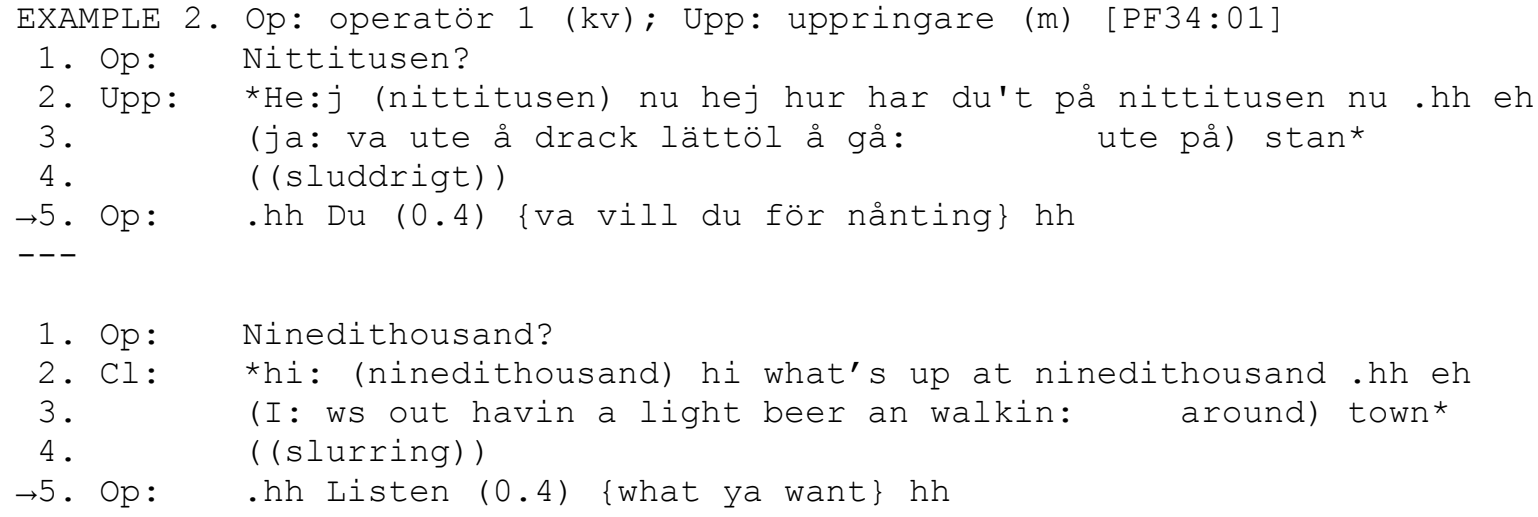

The operator's receipt in line 5 clearly suggests that the caller's first turn fails to supply a relevant reason for calling the emergency number - nor does it treat the caller's engagement in the talk as indication of an urgent need for help. Indeed, the caller's greetings and "what's up"-inquiries in line 2 form an opening which may be "canonical" (Schegloff, 1986) to mundane telephone calls, but one which is typically omitted - for the sake of brevity and focus - in calls for emergency assistance (e.g., Whalen \& Zimmerman, 1987).

Previous literature, along with the above observations, suggests that in dealings with the emergency services, the onus is on the caller to immediately supply a relevant reason for initiating the contact. This can be achieved in different ways and it is the interactional procedures through which the participants arrive at the caller's reason for the encounter that form the topic of our inquiry. Overall, the analysis extends earlier findings on telephone openings in emeregency calls, and points more broadly to some concrete real-world outcomes of communication design work (cf. Aakhus, 2007) in professional settings.

\section{Two corpora of emergency calls}

This article examines two corpora of calls to the Swedish emergency services, SOS-Alarm. The corpora were collected over 20 years apart, roughly ten years before and after the change of the emergency number and the operator protocol. 
The first corpus was collected in 1986 by Bengt Nordberg and associates. It was recorded in one of the smaller emergency centres (hosting 2-4 operators on duty) located in central Sweden. It comprises over 170 calls in total, and entails incoming calls as well as outgoing communications (via phone or radio) from the centre (see also Nordberg, 1999, for more details on setting and corpus). Our interest in this article is with the 86 calls coming in through the emergency number 90000 . Roughly 60 of these calls (70\%) concern medical conditions of varying degrees of emergency, to which the response activities would span from ambulance dispatch to telephone contact with doctor on duty, while the remaining calls deal with fire incidents, suspected crime and various technical malfunctions. As we have already noted, the emergency number 90000 served as the operators' opening move, occasionally in combination with the phrase SOS.

\section{2-corpus}

The second corpus was collected over a period of three years beginning in 2006. It was recorded in different, but similarly sized emergency centre (typically hosting 2-4 operators on duty, but allowing another two to engage in dispatch work when called for), located in a south-central Swedish region, serving a population of just over half a million residents. The corpus comprises over 150 calls incoming through the emergency number 112 from lay members of the public. Like the first corpus, a majority of calls concern medical conditions $(53 \%)$ followed by reports of suspected crime, and other police-relevant incidents (28\%) and fires or other rescue operations (13\%). The remaining $6 \%$ of the corpus comprise matters of no relevance to the emergency services, including such topics as lost pets, God and a variety of more or less straightforward pranks (for a study of prank calls to a child helpline, see Emmison \& Danby, 2007).

\section{Selection, transcription and analysis}

In all, the two corpora hold over 230 audio-recorded calls to the emergency services placed by ordinary members of society. To maintain a degree of homogeneity across the two corpora, our selection of calls prior to analysis of the interaction was guided by the following five criteria: callers were (i) adults, and (ii) lay members of the public (rather than nurses, doctors, ambulance drivers, etc.); (iii) calls came in through the emergency number (90000 or 112), rather than other available service numbers; (iv) operators responded to the summons using the prescribed standard phrase ("ninety thousand" or "SOS one-one-two, what has 
occurred?") and finally, (v) calls were conducted in Swedish. This selection procedure generated 22 calls from the 90000-corpus and 52 calls from the 112-corpus.

Both corpora were transcribed in detail following conversation analytic notations (Jefferson, 2004), slightly adapted to include a range of nonvocal activities such as operator typing sounds and a wide spectrum of background noise, including sounds from pets, radio and television, sirens, etc (see Appendix for transcript key). The 90000-corpus has been transcribed previously by Nordberg and other colleagues. For the purposes of the current analysis, the authors have closely checked the transcripts against the audio recordings to ensure the accuracy and degree of detail to allow for fine-grained analysis of the interaction. Personal, locational and other sensitive information has been altered to protect callers' anonymity.

The data were analysed to reveal the sequential and categorial organisation of the parties' verbal actions in the opening of the call and during the initial phases of the interrogation. Sequential analysis rests on the assumption that social interaction exhibits - as its most elementary property - systematic and orderly features, and that members' actions can be analysed to demonstrate this order with precision. In highlighting features of turn design and their sequential ordering, we aim to explicate the innate organisation of the opening exchanges between caller and operator across the two corpora of citizens' calls for emergency assistance.

\section{Analysis}

\section{0}

We begin the analysis by showing how callers organise their first turns at talk, focusing on the variation that we find in the 90000 -corpus. As a next analytic step, we examine how the parties continue to further unpack the reason for the call, which typically occurs in the immediately next sequential position.

Reason for calling - first turn requests 
Whalen \& Zimmerman (1987) pointed out that callers to the emergency services typically supply their reason for the call by requesting some form of assistance or by producing a report of a troublesome event. We see this in the following extracts:

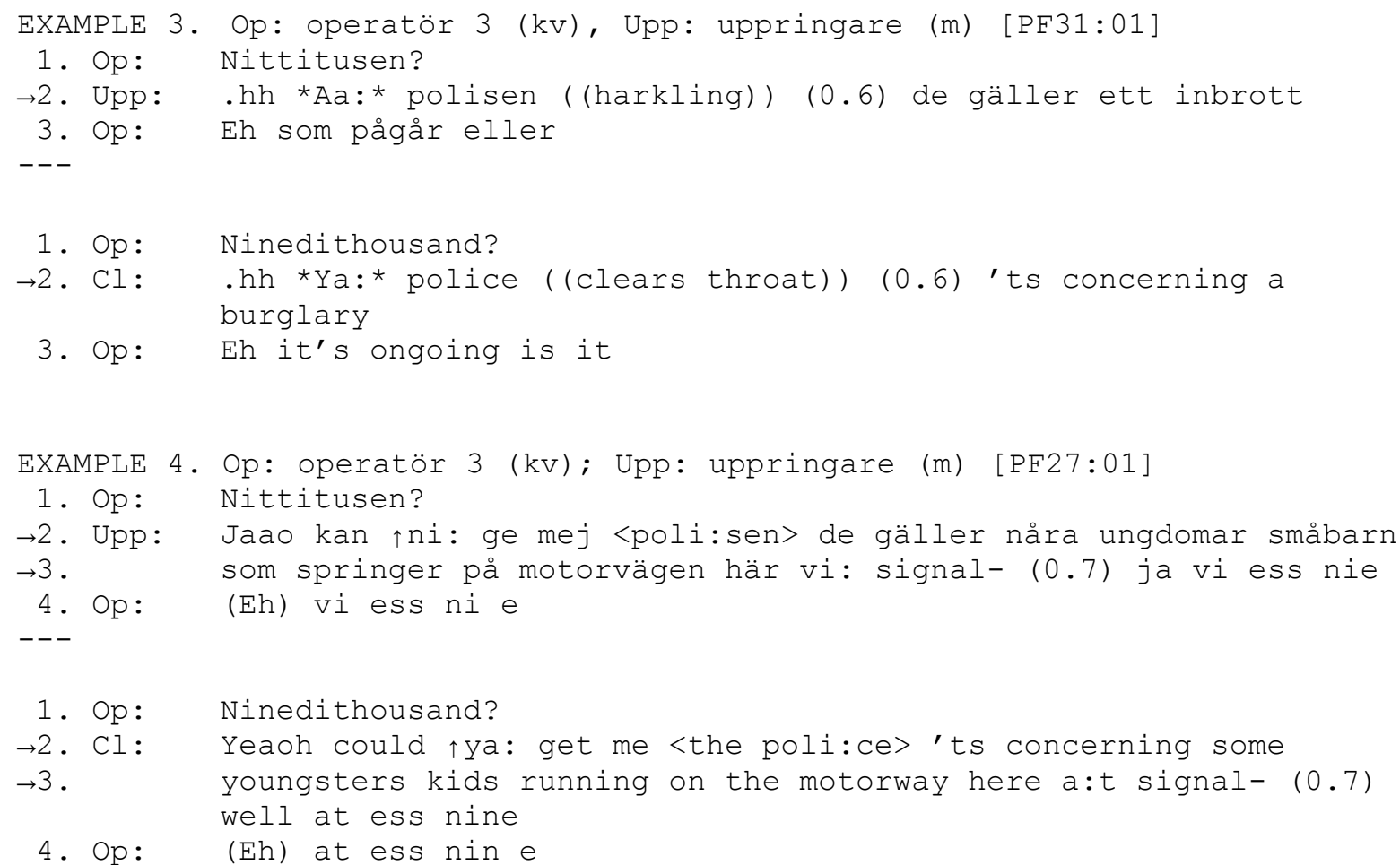

In the examples above, the callers describe in their first turns the troublesome events leading up to the call. In both examples, however, the reports comprise callers' second actions, following upon a prior request for a particular service (police assistance). We may further note that the causal-topical link between the reports and its preceding request ("its concerning") makes the report hearable as an account for the request - it provides the reason for the call generally, and a rationale for that particular type of request specifically. That is to say, the descriptions of the events leading up to the call are not produced as standalone reasons for calling the emergency services - what they do is render a particular meaning to the requests.

Initial caller turns containing event descriptions are not very frequent in the 90000-corpus, accounting for less than $20 \%$ of the calls. When they do occur, they tend to be produced as accounts for an immediately prior request. In this corpus, the most common type of response to the operator's opening phrase comprises a request for a specific service, often combined with a destination for the requested assistance: 


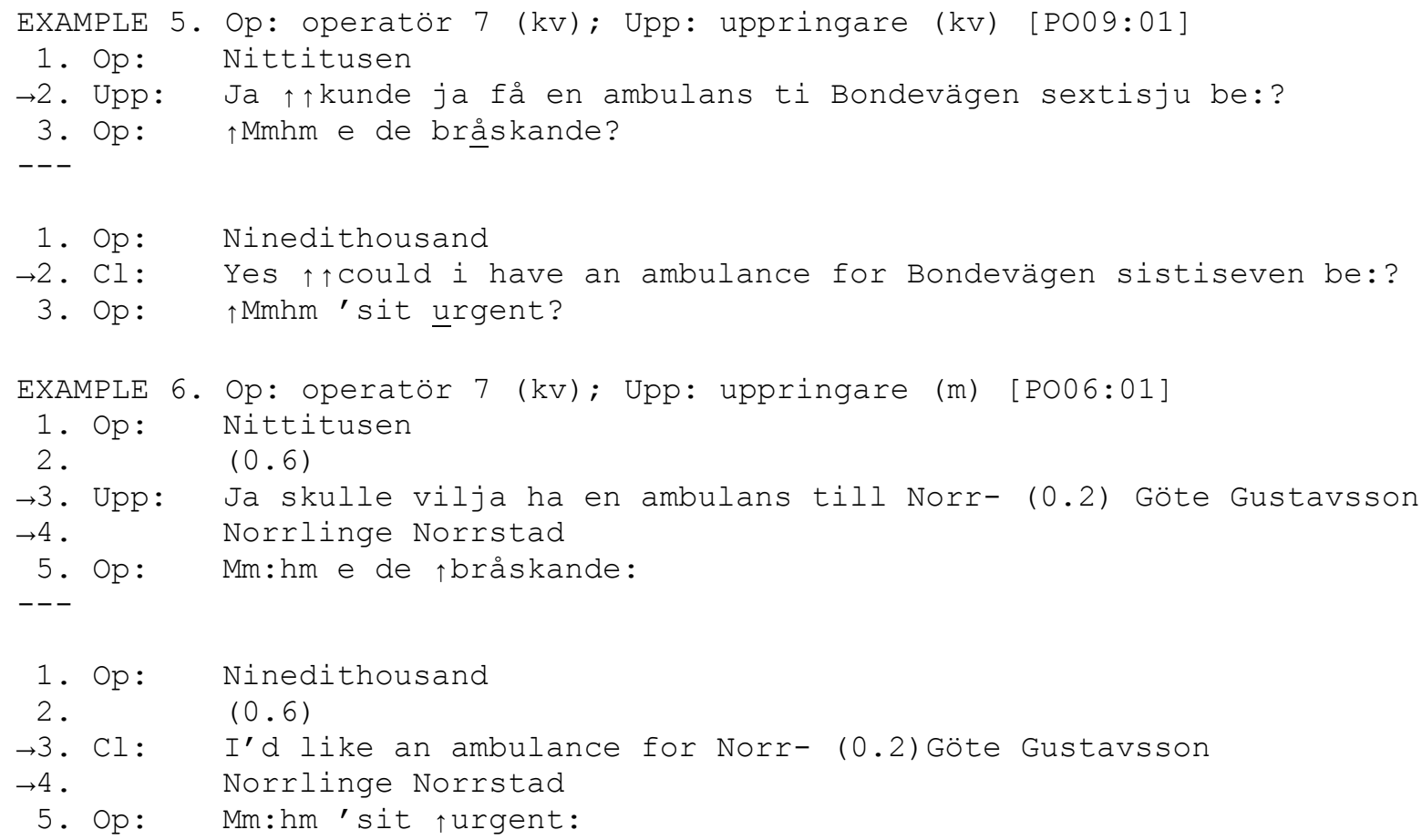

In example 5, the caller sets off by acknowledging the operator's identification (Whalen \& Zimmerman, 1987, see also Garcia \& Parmer, 1999, for a discussion of the local relevance of such acknowledgments). This acknowledgement is absent in example 6, in which the caller notably produces his first turn after a slight delay. Both examples show the callers requesting paramedic assistance, then proceeding to specify a destination for the ambulance. In contrast to examples 1 and 2, the operators' acknowledging receipts of the callers' turns show that actions formatted as request for assistance + location satisfy, for all current intents and purposes, the expectation on the caller to supply a reason for calling the emergency services.

There is some variation to this procedure. For instance, the caller's request for a service and formulation of location may take the reverse order:

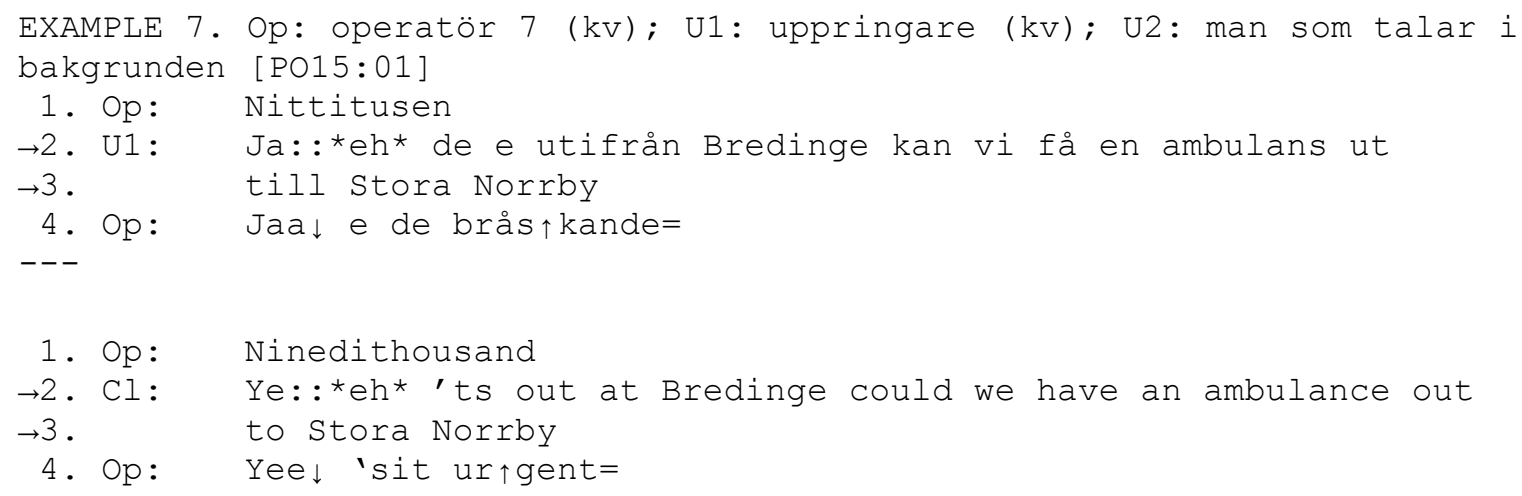


Here, the caller starts out by acknowledging the identification of the emergency services, then moving on to first specify the location and then produce a request for an ambulance.

Occasionally, the caller will just request a particular service, without producing a locational formulation:

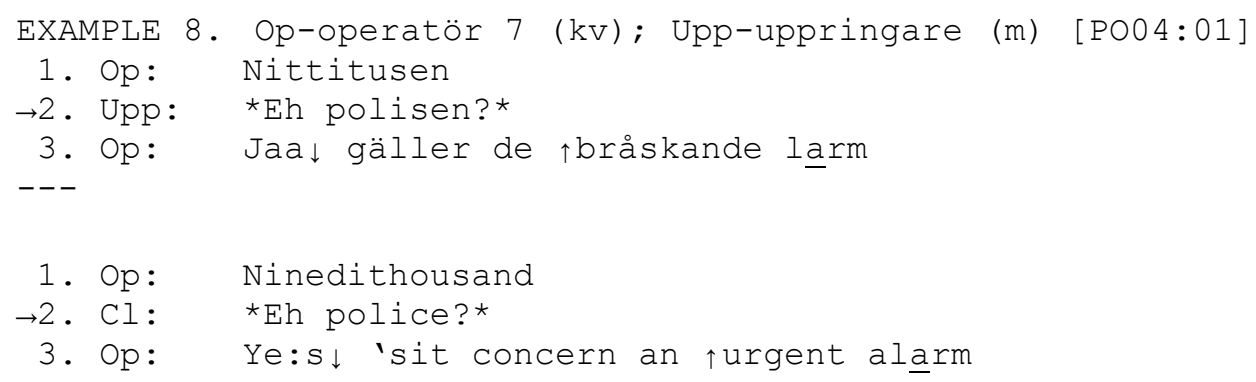

Common to extracts 5-8 is that the operator accepts the first turn of the caller as satisfactorily displaying her/his business with the emergency services. The caller's offering of a reason for the call is thus treated as adequate for the interrogation to continue, and is typically accomplished as part of the operator's second turn in the call. There are some exceptions to this as seen in the two extracts below:

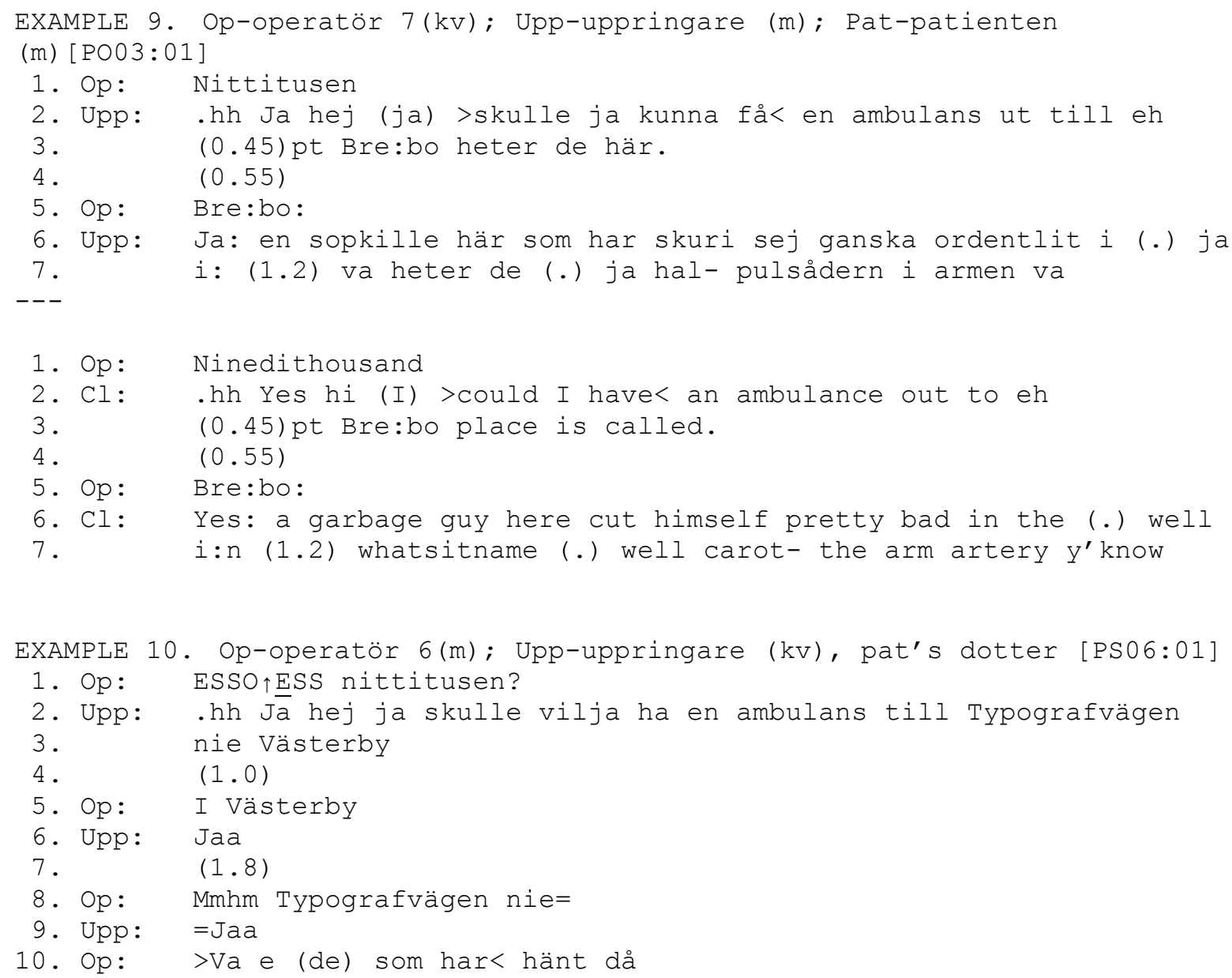




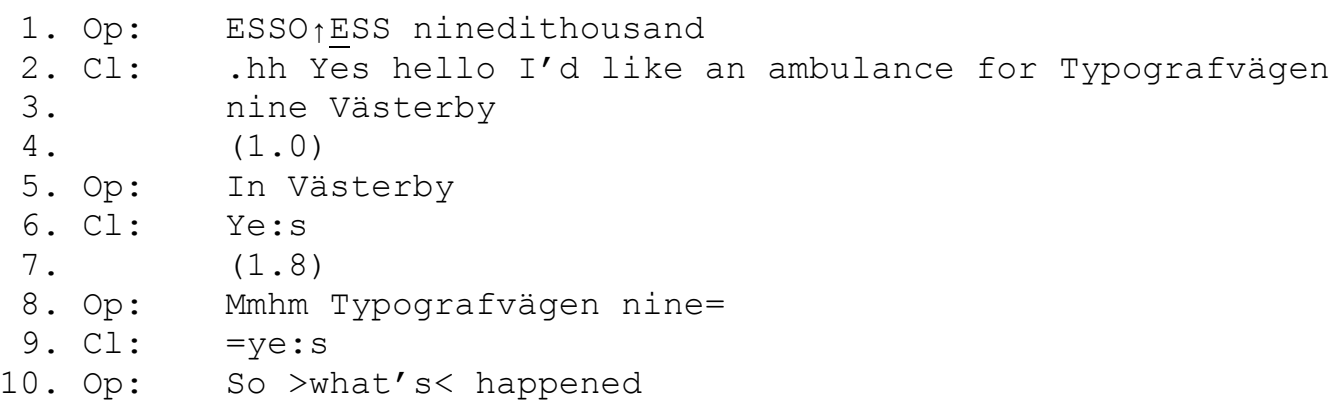

Both examples show the operator initiating a clarification of (parts of) the location offered by the caller. Note that by attending to the specifics of the callers' location formulations, operators are acknowledging the request + location format as an institutionally relevant way of presenting the caller's business. We conclude this section by showing a slightly deviant example, in which the caller gives his address but fails to ask for assistance:

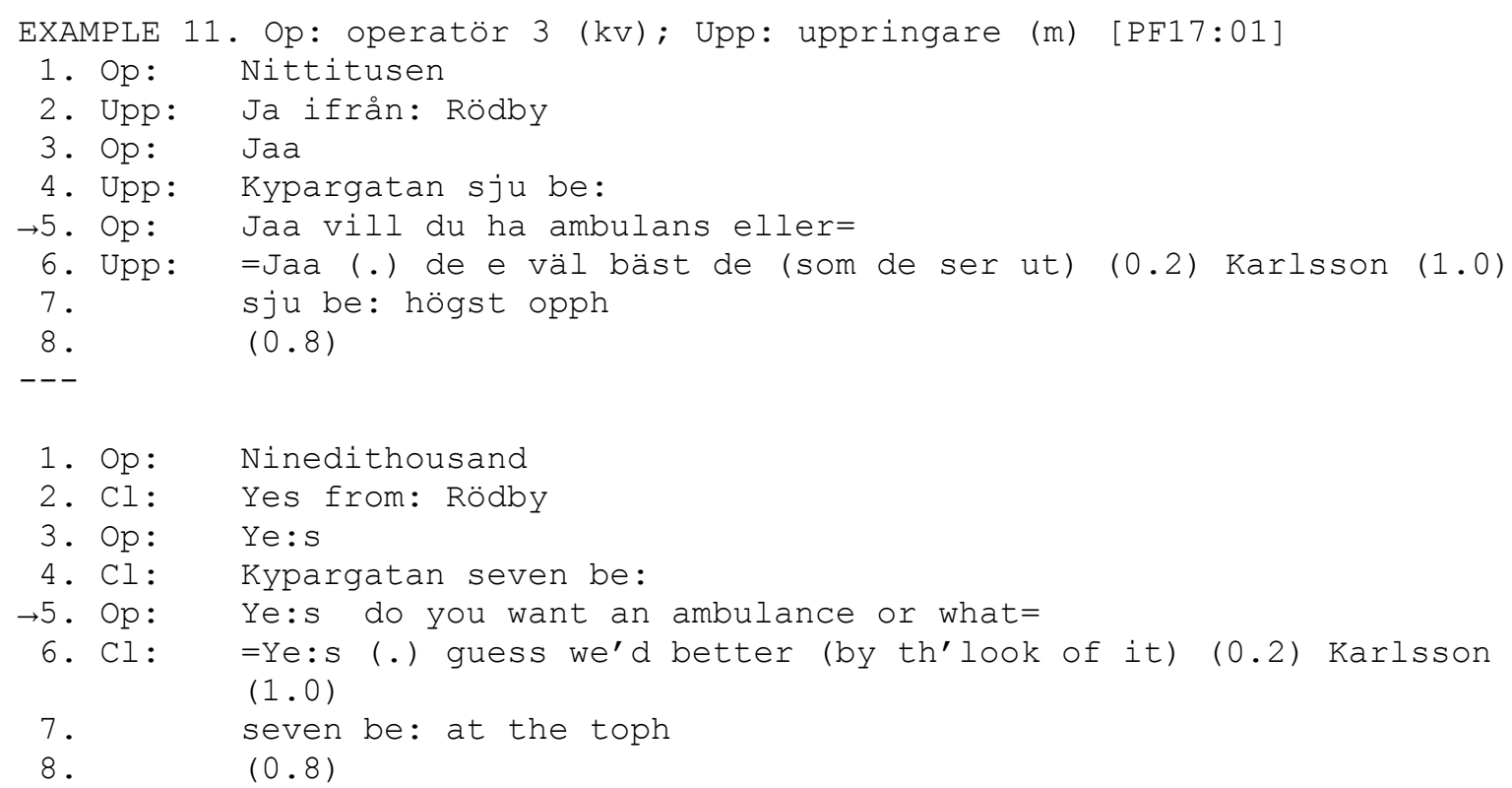

In line 2, the caller starts off with a general locational formulation, identifying the village of Rödby. This is acknowledged by the operator and the caller proceeds to narrow down the location to a street address (line 4). The operator's following turn "ye::s do you want an ambulance or what" identifies the previous exchange as problematic. More specifically, it points to the absence of a proper reason for calling and proffers a candidate request for an ambulance, which the caller readily confirms in line 6 . This candidate request not merely shows the operator's orientation towards the inadequacy, or incompleteness, of caller's "first topic"; it also reveals his expectation as to the specific format for the packaging of the caller's 
reason for the call. In a nutshell, it shows that operators expect callers to request certain types of assistance as part of their first turn in the opening of the call.

\section{Probing the reason for calling}

We have established that following upon the operators' opening line "ninetythousand?", callers typically request a specific form of assistance. Such reports may be combined with other actions, such as locational formulations or, more infrequently, reports of trouble that serve as accounts for the request. The operators' responses to these turns show that such requests comprise an orderly, and locally normative, way of presenting the caller's business.

We now proceed to examine the continuation of the interrogations. Unless a clarification sequence is inserted to deal with parts of the caller's first turn (as in examples 9-10), a typical continuation at this point in the call is for the operator to inquire about the urgency of the matter, as we can see in extracts 5-8 above $\mathrm{v}^{\mathrm{v}}$. Let us consider this questioning practice and the type of caller actions that they engender:

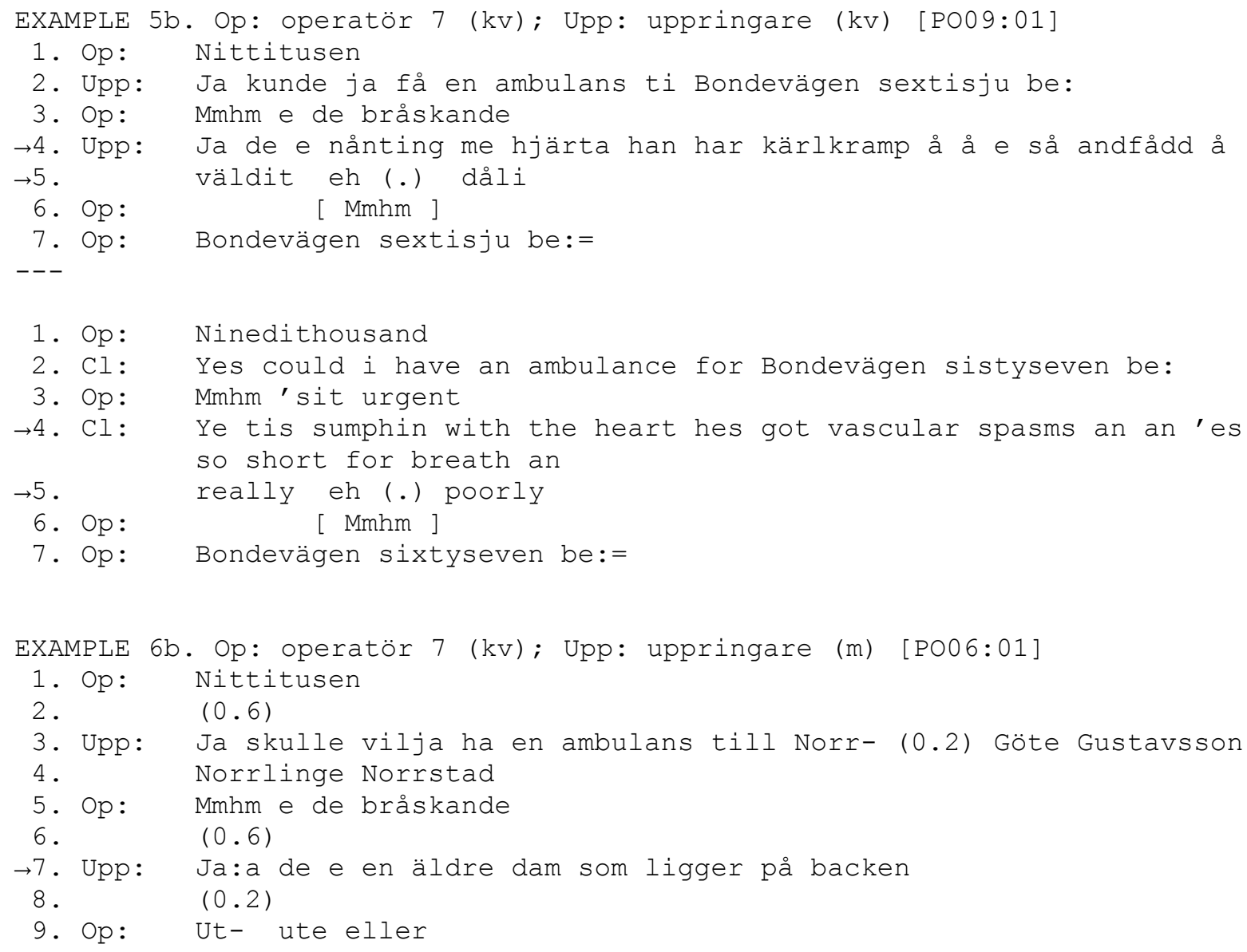




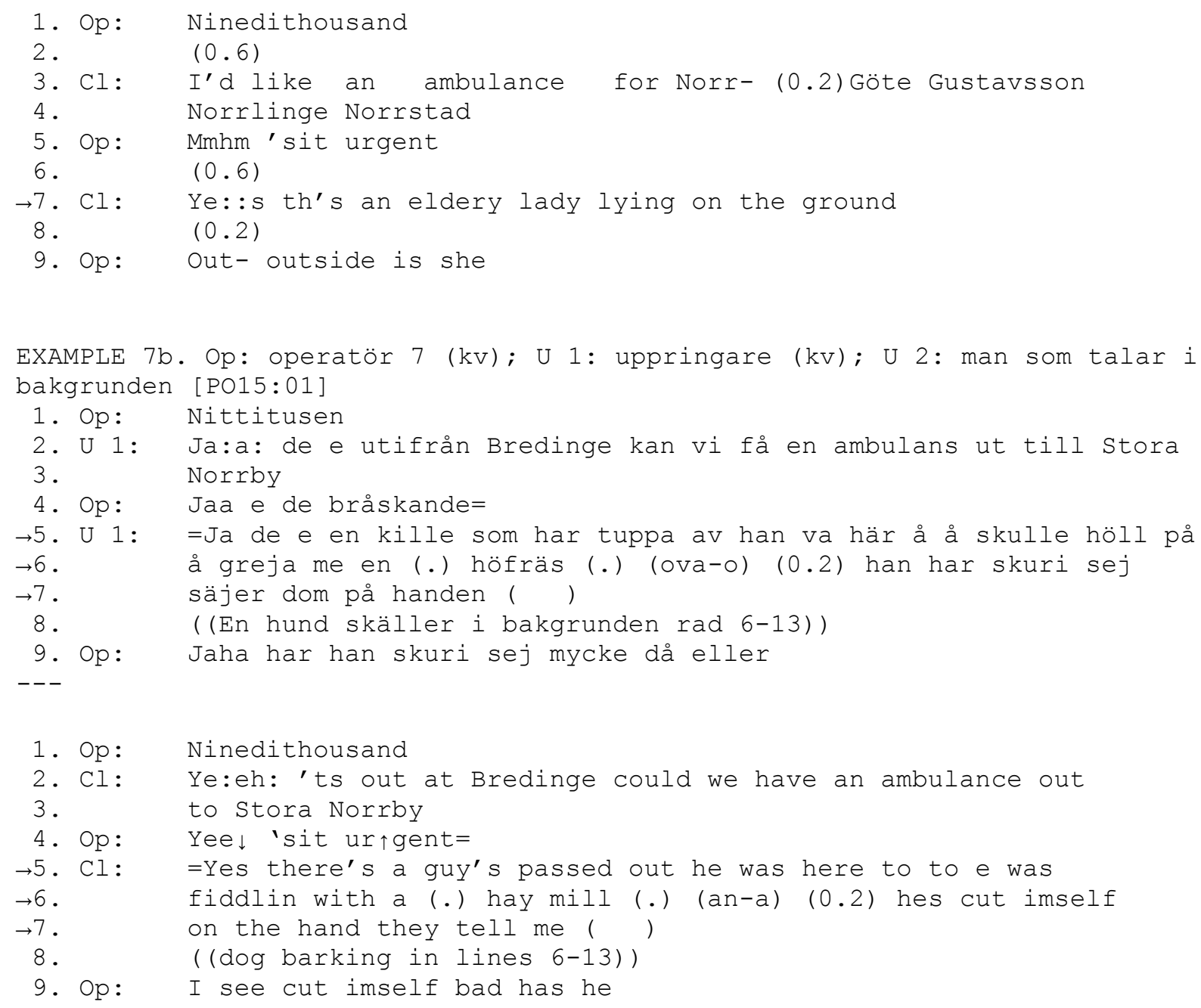

In extracts $5 b-8 b$, the operator responds by acknowledging (through "mhm"-tokens in example 5b-6b and in lexicalised form, "yes", in 7b-8b) the caller's first turn, then immediately proceeding to ask about the urgency of the incident. One interesting feature of these questions is that they invariably elicit from the callers' reports of the trouble, rather than 
simple acknowledgments of urgency. Hence, in response to the operator querying the urgency of caller's business, the callers offer descriptions of the troublesome event; descriptions that account for the call as an emergency call. That is to say, the operator's question is routinely treated by the callers as probing the relevance of their business for the emergency services. One slightly deviant case clearly illustrates this point:

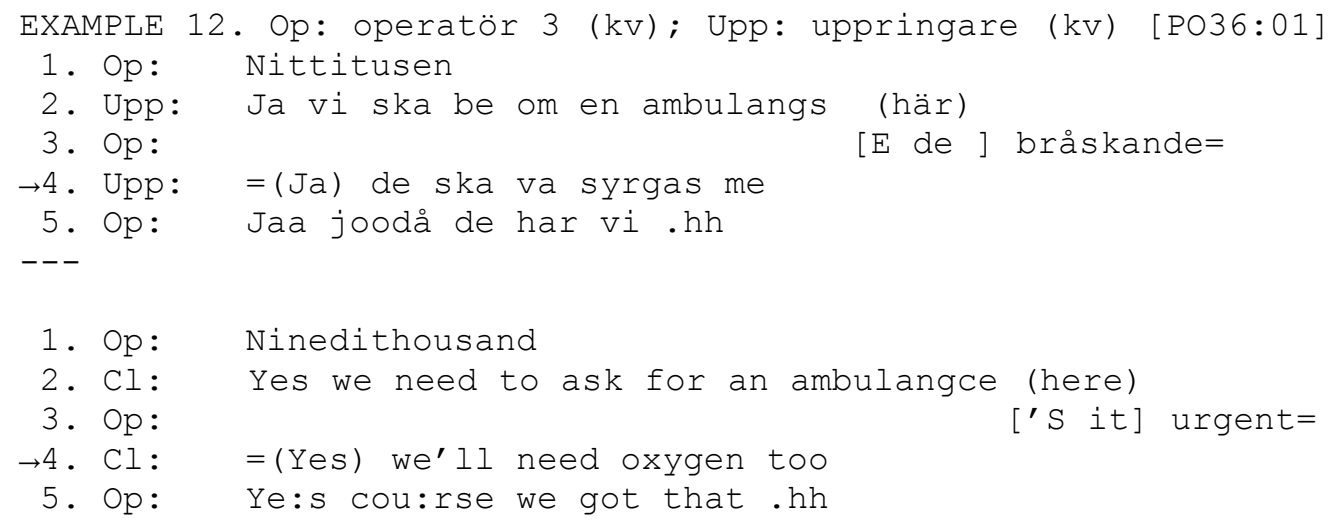

In response to the operator's question about urgency (line 3), the caller confirms and adds that the patient will need oxygen. By stressing the need for oxygen, the caller categorically rules out any other means of transportation that the one she had requested. Hence, she is accountably hearing the operator's question in line 3 as probing the institutional relevance of the event that led the caller to ask for emergency assistance.

In the analysis of call openings in our first corpus, we have shown that upon the operators' identification of the emergency services in the opening announcement of the emergency number 90000 , callers typically use their first turn to request a specific type of emergency assistance. In fact, inspection of a deviant case revealed that requests for help, whether solitary or combined with other actions, such as acknowledgements of the identification, location formulations or accounts for the request, form part of the operators' expectations concerning what the callers ought to be doing in their very first turn at talk. In the typical case, these formulations of callers' business are then elaborated through descriptions of the troublesome event elicited by the operators' routine question about the urgency of the matter. We now move on to consider our second corpus, targeting the same sequential positions in the opening interactions of the post 1996 calls to the emergency services. 
If requesting assistance formed the normative and single most common way for callers to present their business in the 90000 corpus, then the 112 corpus is characterised by their general infrequence as well as dispreference. We begin this part of the analysis by discussing the operators' standard opening phrase, then examine in brief the responses that it routinely engenders. We conclude the analysis with a call in which the pattern is broken, revealing a very different, yet equally normative, order underlying the organisation of call openings in this corpus. Both the normative cases and the deviant example demonstrate the parties' monitoring of and orientation to the sequential properties of action, as well as their institutional relevance in the context of calls for emergency assistance.

\section{Reason for calling - first turn reports}

In our discussion of the previous corpus we pointed out that the operators' opening line "ninety thousand" works as an institutional identification for the emergency services. We further pointed out that this opening partitions the interlocutors along two mutually constitutive categories: the operator's self-identification as "help provider" automatically invokes an identity for the caller as "help seeker".

Turning now to our second corpus, we note that the standard phrase by which the operators answer the summons also contains an identification combining the term SOS with the emergency number, "SOS One One Two". In addition, the opening line contains a second part; a direct inquiry about the event that has led the caller to dial the emergency number (cf. Cromdal, et al., 2008, for a more thorough discussion of this opening phrase). Consider the opening turns in examples 13 and 14 below:

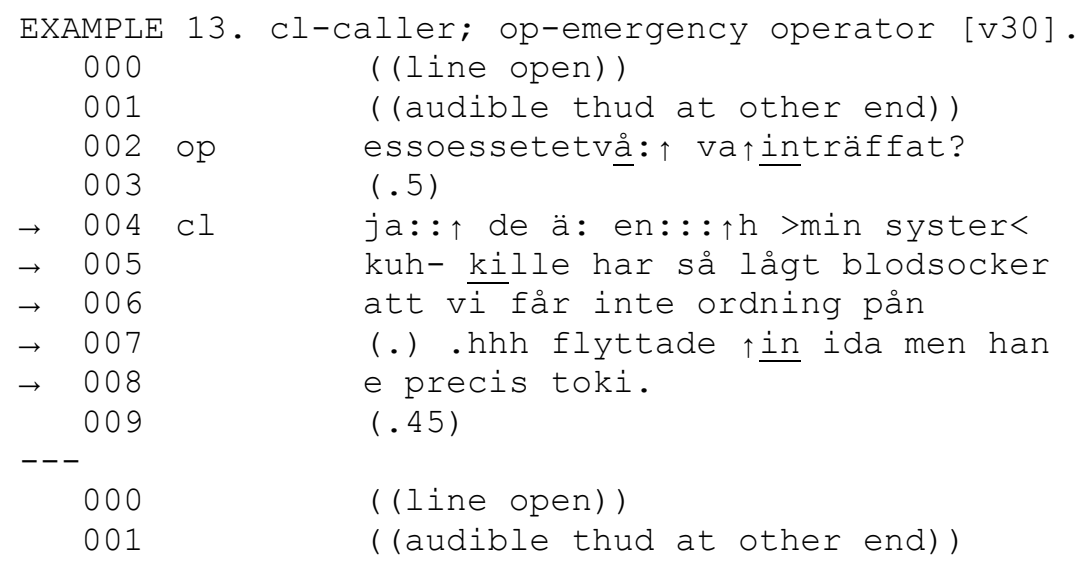




$\begin{array}{lll} & 002 \text { op } & \text { essoessoneonetwo }: \uparrow \text { whs } \uparrow \text { occurred? } \\ & 003 & (.5) \\ \rightarrow & 004 \text { cl } & \text { ye: }: \uparrow s^{\prime} \text { tis: e: : : } \uparrow \text { h >my sister< } \\ \rightarrow \quad 005 & & \text { buh- boyfriend's bloodsugar got so low } \\ \rightarrow \quad 006 & \text { that we can't handle im } \\ \rightarrow \quad 007 & (.) \text {.hhh moved } \uparrow \text { in tday but 'es } \\ \rightarrow \quad 008 & \text { downright bonkers. } \\ & 009 & (.45)\end{array}$

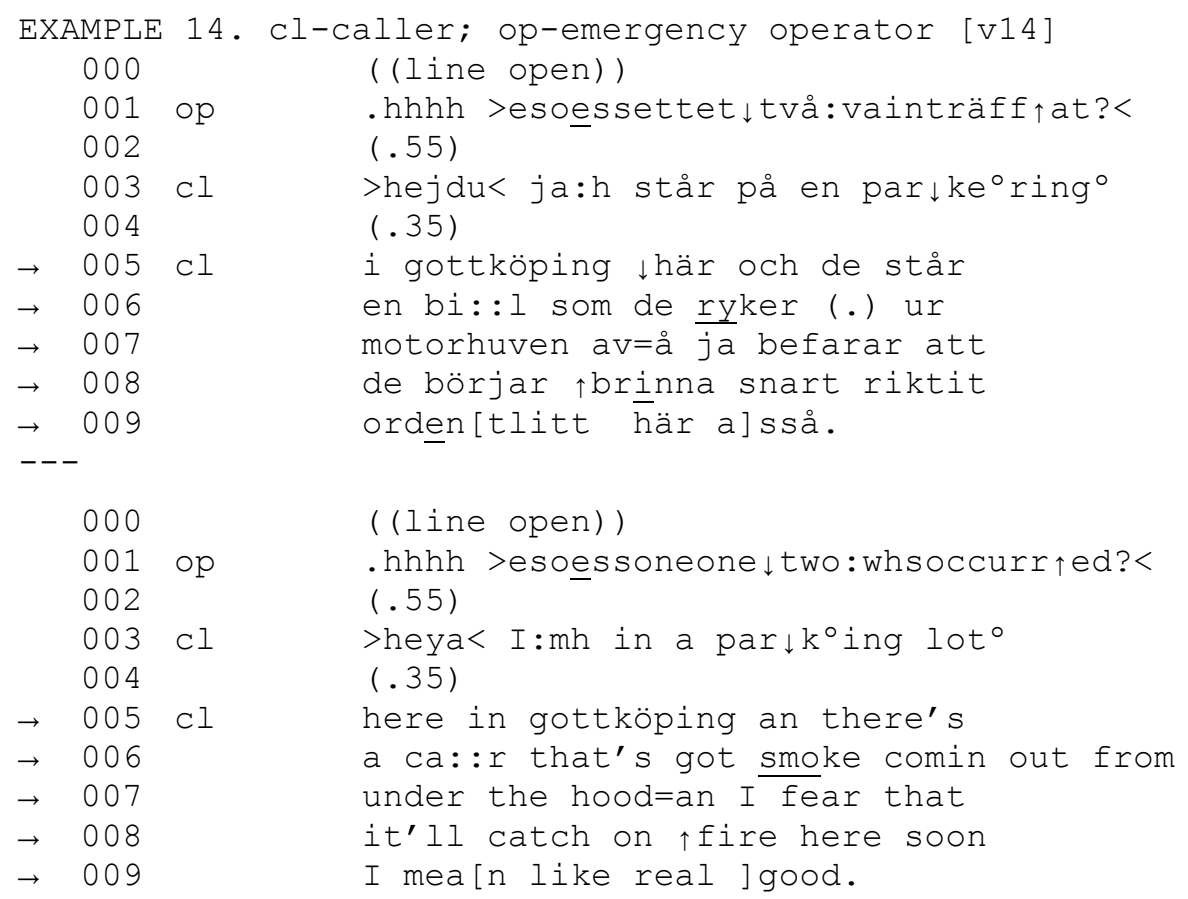

The two examples above show the callers first responding to operators' opening by acknowledging the identification of the emergency line. They then describe, in the same turn, the event that they feel requires emergency assistance. In example 13, the caller builds up, with some effort, a narration about her sister's (presumably) diabetic boyfriend going “downright bonkers" due to low blood sugar. In example 14, the caller's acknowledgement takes the lexical form of a greeting. It is swiftly produced, and does not leave a slot for the operator to return the greeting. Such design allows the caller to acknowledge the identification without initiating a greeting sequence. The next action of the caller in example 14 is a locational formulation, followed by a description of a car catching on fire - though it could be argued, of course, that locating the fire to a parking lot is also part of the description of the imminent danger (other cars in the parking lot may be a worry), rather than a "pure" locational formulation.

Whereas in example 13, the caller's report contains clues that she (and her sister) may be in personal danger, in example 14 the caller's report entails his declaration of a personal worry 
about other people's property, thus taking on the format of a "good citizen's call" (Cromdal, et al., 2008; Eglin \& Widemann, 1979; Turner, 1969). These and other differences aside, the point we want to raise here is that the reason for the call in the first turns takes the shape of a report of some specific instance of trouble. In other words, both callers show sensitivity to the operators' routine opening, delivering actions that are conditionally relevant to the query concerning what had "occurred".

Our next example adds some variation to this pattern:

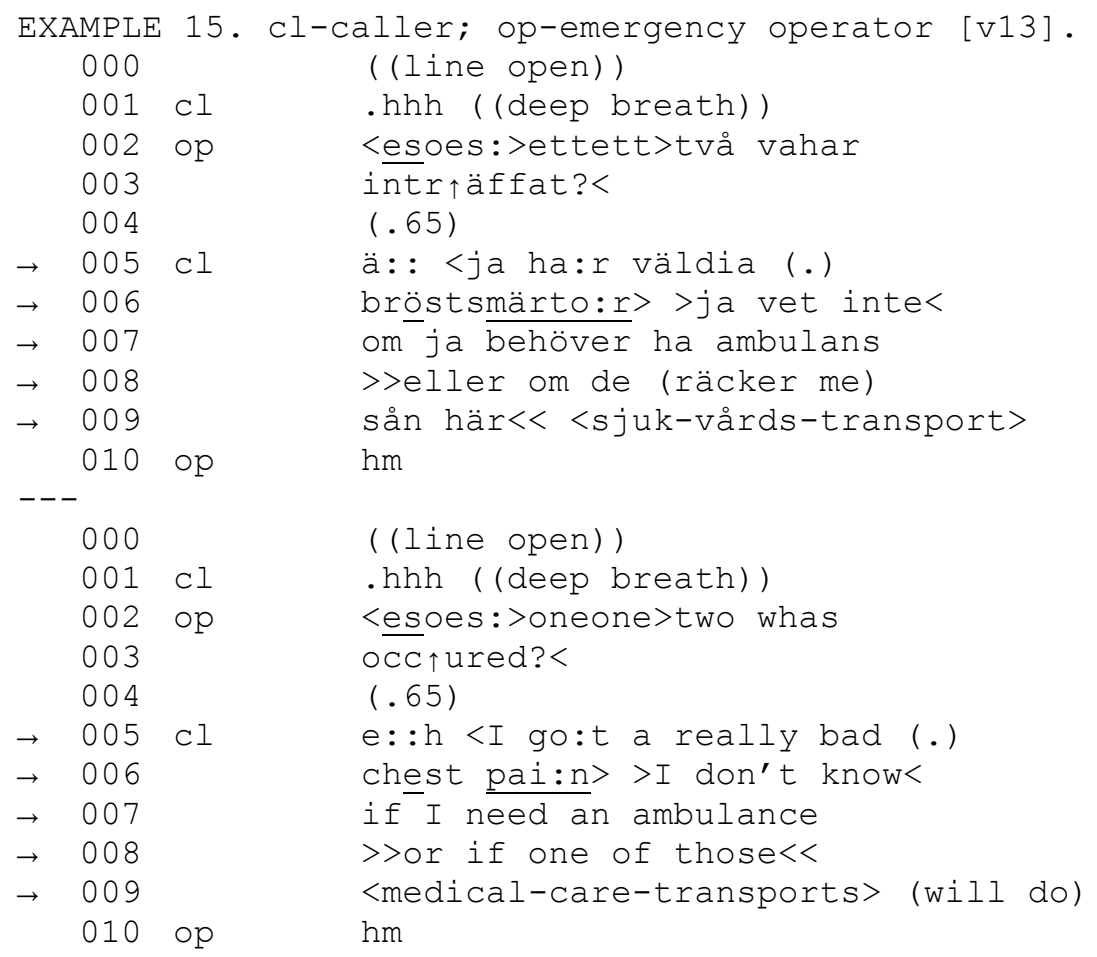

Following upon the operator's opening, there is a brief pause, then the caller starts off with a hesitation token "e::h" (line 5). Compared to the previous examples (13 and 14), this is a rather different way of starting out, indicating thus far in the exchange that the caller is not entirely certain that she has called the right place. She then describes her symptom "a really bad chest pain", and explains that she does not know what type of medical transportation to ask for.

Clearly, this caller is unsure whether she should be calling the emergency services, and she uses her first turn to explicitly present her uncertainty about her own need for assistance. While this example clearly differs from the two above with regard to the caller's displayed epistemic stance (cf. Drew, 1991; Heritage \& Raymond, 2005), her actions are designed - 
through the initial offering of a symptom - to satisfy the operator's opening query ("> whas occ $\uparrow$ urred? $<")$ in lines 2-3. With this initial account of the opening actions of operators and callers, we now move on to consider the exchanges immediately following in these calls.

\section{Reason for calling - working out the details}

In our first corpus, we noted that, following the callers' request for emergency assistance, the operators routinely inquired about the urgency of the matter; a questioning practice that typically elicited a report of the troublesome event, rather than a plain confirmation of urgency. In the second corpus - in which callers observably attend to the sequential implicature of the operators" opening phrase, producing a report of "what has occurred" in their first turns - we find a different trajectory of action, as illustrated by examples $13 \mathrm{~b}$ through $15 \mathrm{~b}$ below:

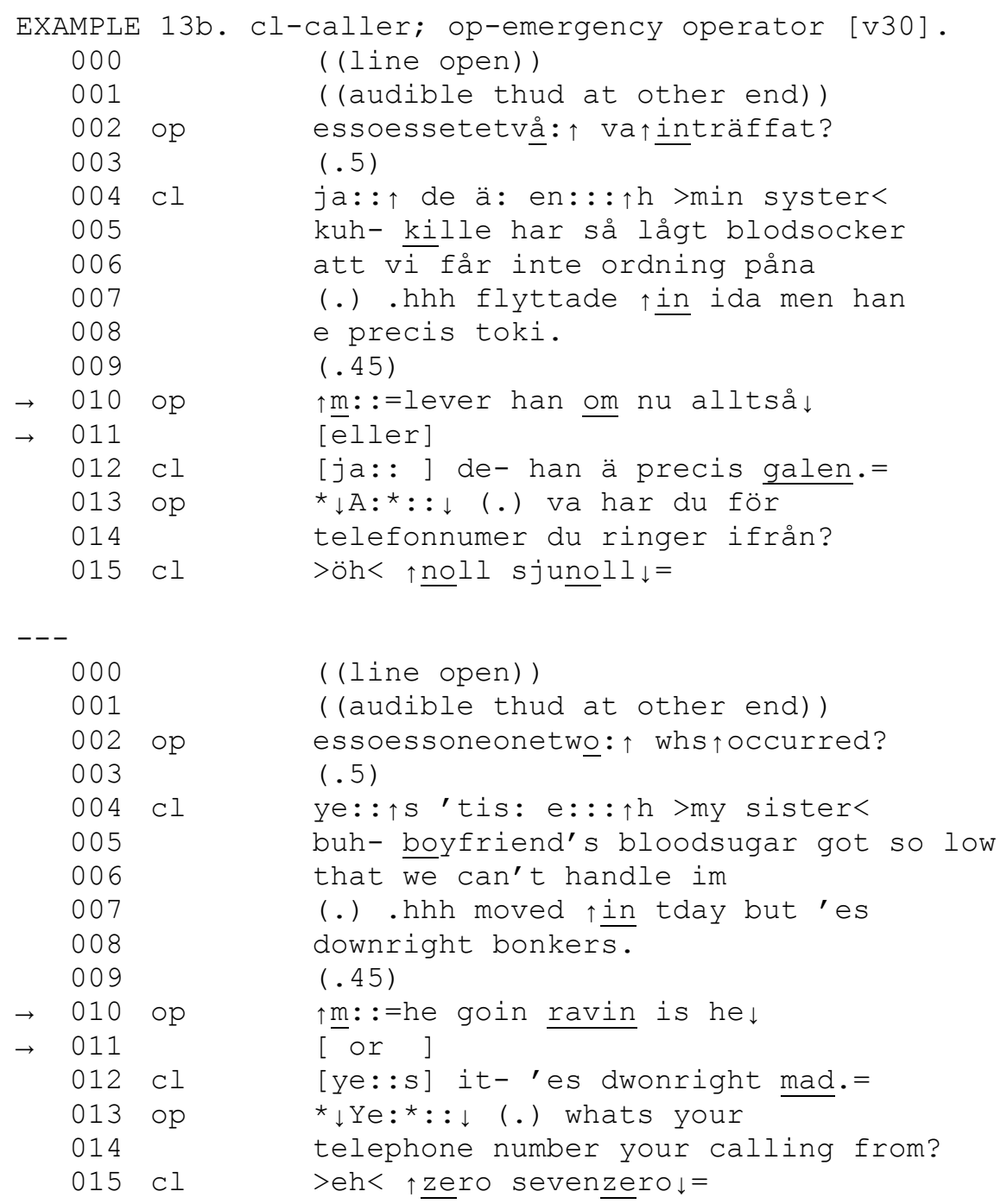




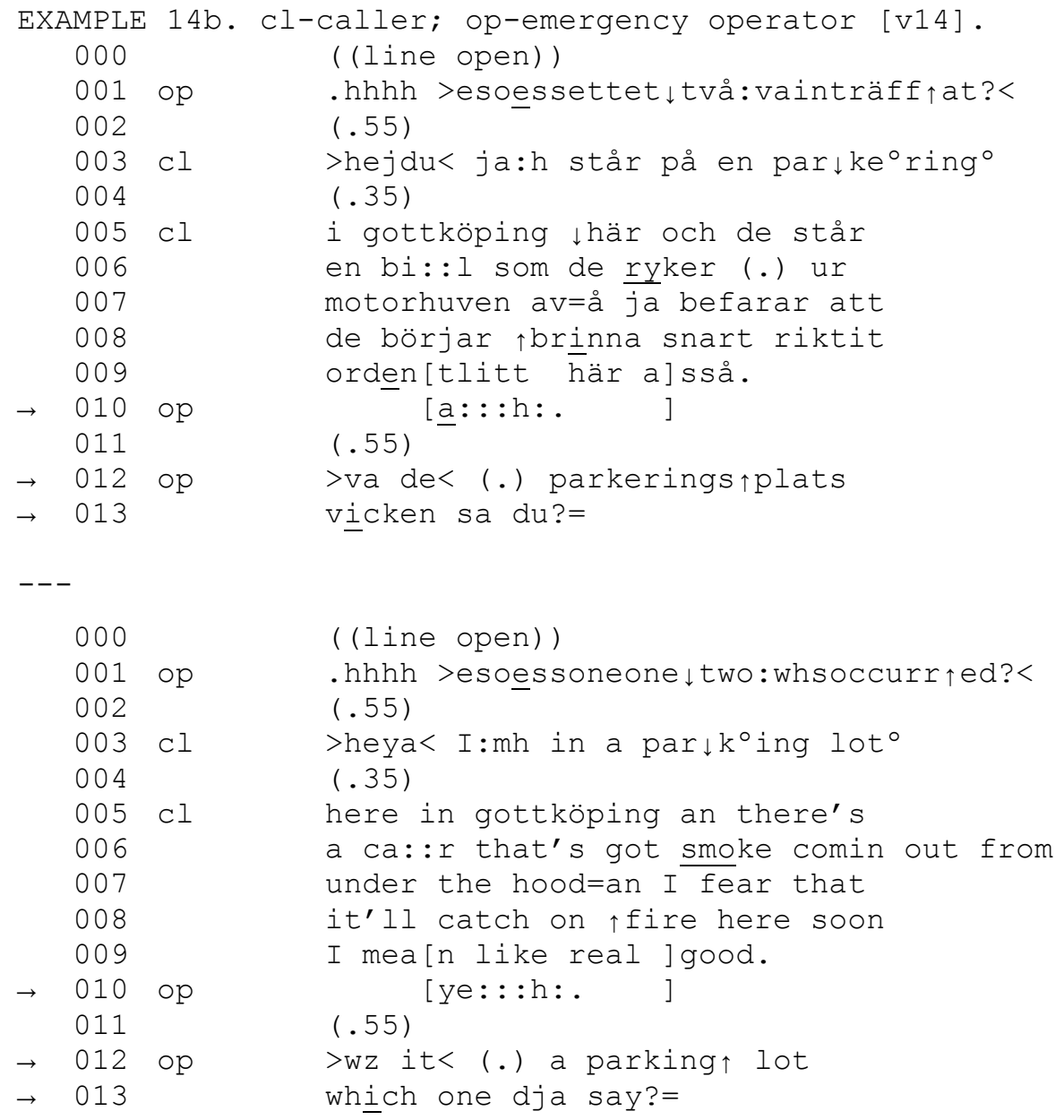




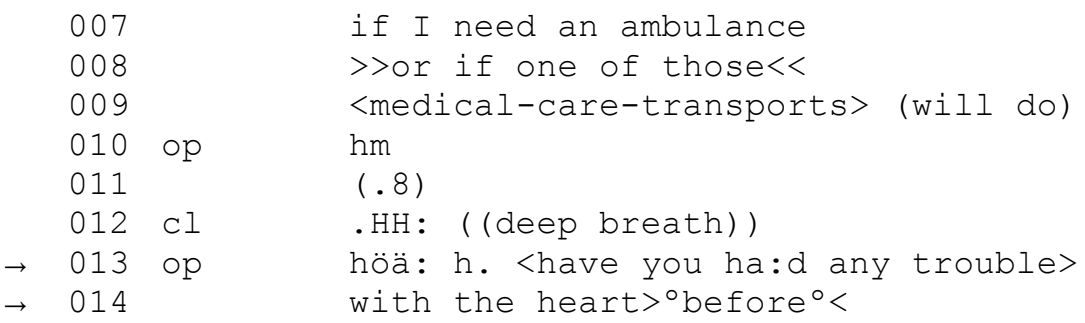

In example 13b, the operator begins to unpack (lines 10-11) the caller's glossing of her sister's boyfriend as "downright bonkers"; example 14b shows the operator trying to solicit a more accurate location from the caller (lines 12-13) and, finally, in example 15b the operator commences a diagnostic interview (lines 13-14) concerning the caller's medical history.

In contrast to the routine question concerning the urgency of the caller's business (cf. Excerpts $5 \mathrm{~b}$ through $8 \mathrm{~b}$ ), the operators in the present corpus attend to the details of the callers' initial reports, using their second turn slots to tease out further information relevant for deciding what (if any) services should be dispatched. In sequential terms, the operators treat the callers" organisation of "first topic" as relevant to their opening phrase - as an institutionally "workable" first move towards an incipient series of professional decisions.

\section{A deviant case}

We have noted that the operators' opening phrase in the second corpus has a more narrow sequential implicature. This is the case in slightly over $80 \%$ of the calls, which means that there is some variation that needs to be taken into analytic account. In our final example, the caller responds to the opening query with a request for paramedic assistance followed by a destination - a format that we found characteristic of the 90000-corpus:

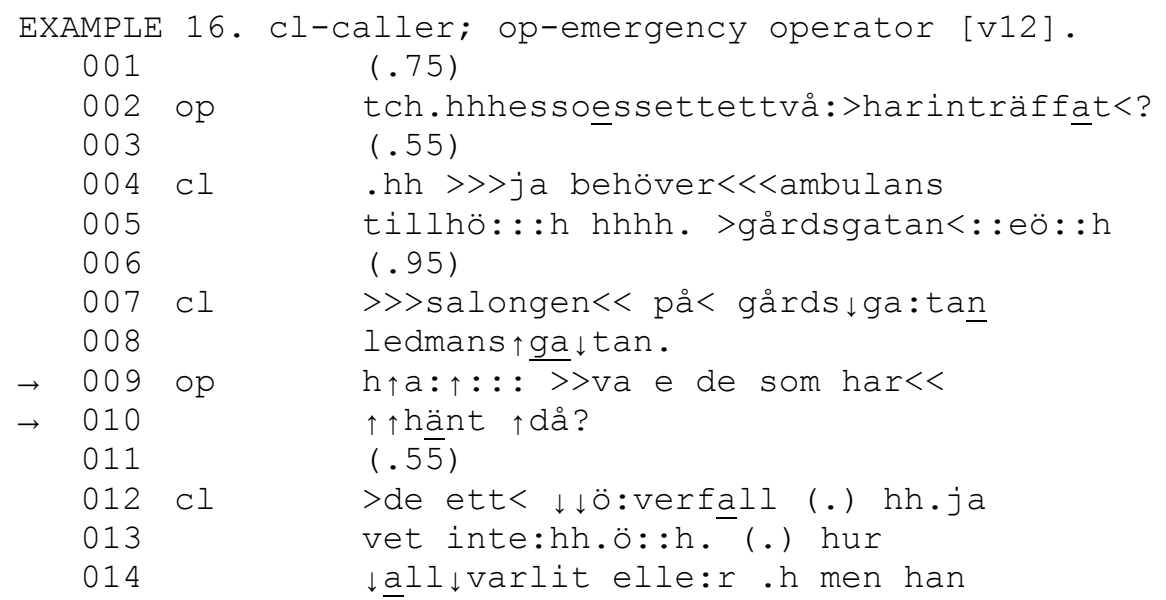




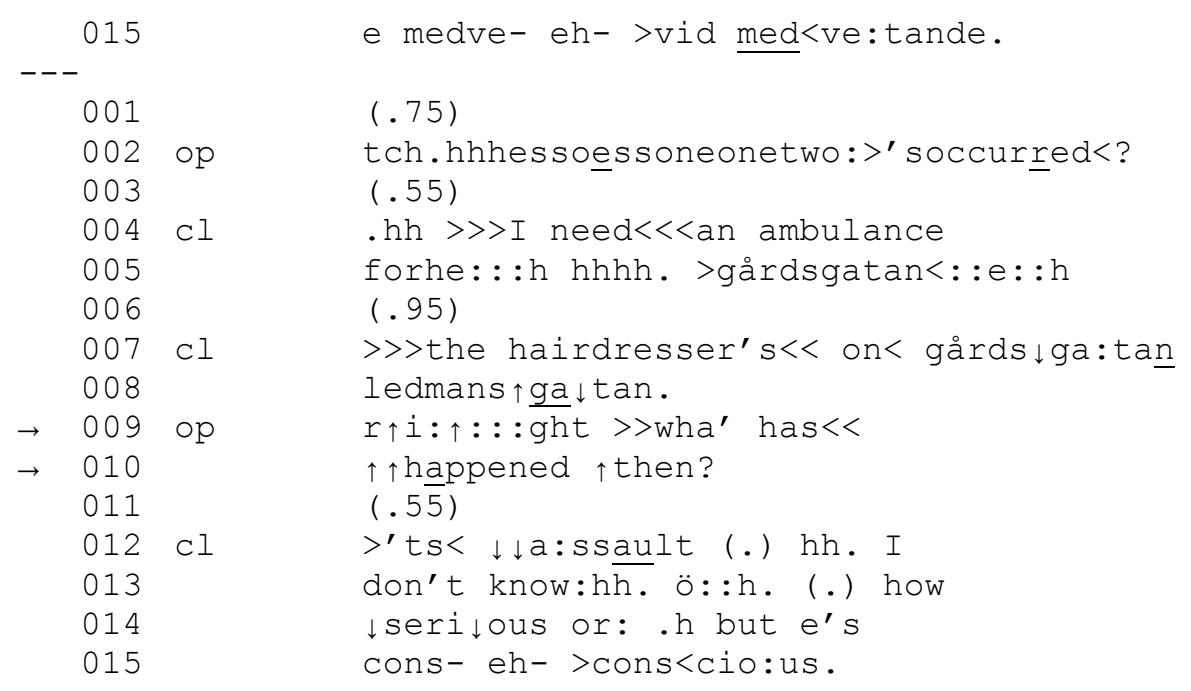

Upon the caller's request for an ambulance and locational formulation in lines 4-5 follows a pause, possibly resulting from the stretched vowel sounds "eö::h" placed at the end of the caller's turn indicating that the caller is planning for a continuation of the turn ("doing thinking"). As it turns out, the continuation entails a more accurate destination for the requested ambulance (lines7-8). The operator responds in lines 9-10 with a heavily prosodically marked receipt of the caller's turn, and then asks the caller what has happened. Let us stop to consider the interactional workings of this action.

Our first observation is that the prosodic stretch of the operator's initial receipt token contrasts with the pace of the caller's turn, which contains several fast-paced elements. A possible interpretation is that, insofar as the caller is displaying urgency by temporal-prosodic means, the operator is, at this point, not aligning with the caller. Second, and arguably, the operator's question "what has happened" (lines 9-10) is hearably a paraphrase of her initial query "what has occurred". This hearability is provided for by the terminal tag "då" ("then"), which, together with the prosodic marking of the word "happened", displays the operator's orientation that something has gone amiss in the caller's turn and locates the notably absent information to the part dealing with what has "happened". Hence, the operator's turn is designed to manifest the receipt of the caller's actions, and to indicate at the same time that its institutional relevance remains to be established. Centrally, it locates the incident - the "occurrence-happening" - as the procedurally crucial, yet unavailable, information in the caller's first turn.

In light of this, the caller's response can be heard as oriented to the previous omitment: he starts off with a gloss (“överfall”; eng: "assault”) - a strictly legal term in Swedish - that 
provides a very efficient description of the situation. He then follows up with some crucial information regarding the medical status of the victim. The caller's second turn at talk displays his understanding of just what type of information the operator has been pursuing from the outset. More generally, this excerpt shows how operators routinely monitor callers' first turns for descriptions of events, rather than requests for assistance, and how they orient to the absence of such reports as an accountable act, an omission in need of a prompt remedy.

\section{Conclusions and discussion}

Calls for emergency assistance provide concrete instances of citizens' dependence and claims on society's official services and resources. In analysing such calls to highlight the internal organisation of the parties' actions, we shed light on the "practical oral production" of one of society's institutions (Watson \& Sharrock, 1990, p. 25). The meeting between professional operators - who are responsible for dispatching and otherwise managing the services available in productive and logistically efficient ways (e.g., Artman \& Waern, 1999; Ikeya, 2003; Normark, 2002) - with lay members of the public, who unlike the operators often have first-hand information of the incident, has drawn some previous research attention. In particular, the opening moves of such conversations have been identified as crucial points in the interaction, bearing in consequential ways on the continuation of the call (e.g., Garcia \& Parmer, 1999; Wakin \& Zimmerman, 1999) and, therefore, on the potential success of rescue operations.

In this article, we have been concerned to examine the organisation of opening interactions across two sets of calls to the Swedish emergency services. Our access to the two collections allowed for a historical-comparative analysis of persons engaging in similar forms of institutional business across distinct interactional settings. In this sense, we were able to locate some concrete outcomes of communication design work aimed at enhancing the professional practice of SOS Alarm emergency operators (cf. Aakhus, 1997; see also Pettersson, 2001, 2004 , for discussions of other design aspects of emergency operators' work). More specifically, the analysis yielded two distinct, institutionalised procedures for responding to the summons as well as their equally distinct consequences for the ensuing actions of the callers. 
The presentation of the caller's business upon the standalone identification of the emergency services "Ninety Thousand" and "SOS Ninety Thousand" typically entailed a request component, often combined with a locational formulation. We noted that both parties oriented to the normative status of a request for a specific form of assistance or service - any omissions of this element were noted, highlighted and rectified in the immediately ensuing talk. We also noted that, at this point in the call, formulations or reports of trouble were neither commonly produced by callers, nor expected by operators. When such reports did occur as part of the callers' first topics, they served as accounts for the request for help. Instead, in the 90000-corpus callers' reports of trouble were solicited through operators' inquiries about the urgency of the matter, which thereby initiated the interrogation series in the call.

As a matter of contrast, in the operators' routine opening phrase "SOS One One Two, What has occurred?", the retrospective orientation to the summons is interlocked with a prospective orientation (see Schegloff, 1986, 2007 on sequence interlocking) to the next turn. The conditionally relevant next action (Sacks, et al., 1974), and caller's first turn at talk, is a formulation of the incident causing the caller to seek emergency assistance. Wakin \& Zimmerman (1999) observe similar instances ${ }^{\mathrm{vi}}$ of opening lines, interlocking the identification of the service with a progression-oriented request for a specification of a caller's trouble. Such a procedure, they suggest, enables a "short-circuiting" (p. 425) of callers' requests for a specific type of assistance, which turns on the assumption that the very act of calling the emergency number may be treated as a request for help.

In view of the two corpora, the openings in the 112-corpus entail another truncation, where the need for the caller's explicit request for service is normatively deleted through the operator's routine response to the summons. The result, in the normative case, is that the opening of the call is shortened by one sequence and that the interrogative series begins with the very first turn at talk.

However, there seems to be more to this than mere shortening of the introductory exchange by a single conversational sequence. What is being truncated, specifically, is a conversational slot which, as we saw in the 90000-corpus, callers typically use to request specific forms of assistance, such as ambulance, police or fire brigade. Organisationally then, by removing this opportunity and replacing it with a direct question about the nature of the incident, the choice 
of (type and amount of) response units is shifted to the emergency operator ${ }^{\mathrm{vii}}$, and Extract 16 shows how such caller requests are immediately sanctioned. While there may be several organisational reasons why callers' requests for a specific response activity are of limited interest to the work of emergency dispatchers, the main one perhaps concerns most callers' lack of professional knowledge of response work ${ }^{\text {viii }}$. It could be argued that the caller's request for a specific service may inform the operator about the type of emergency at hand. However, our analysis of the callers' first turns across the two corpora suggests that such information is more accurate and rich in detail when delivered in response to an actual query about the incident.

The second action being deleted through the opening procedure of the 112-corpus is the operators' question about the urgency of the need for help. Strictly from the perspective of the institution, it seems plausible to argue, again, that lay callers are no longer offered the opportunity (or burdened with the task and responsibility) to comment on response speed and dispatch-related priorities. Whether or not this was indeed an organisational concern when the call-taking protocol was modified, our analysis suggests that the matter of urgency per se was very swiftly attended to by the callers, who instead used the operator's query to produce preliminary descriptions of the incidents.

In conclusion, through the interlocked design of identification + incident query in the opening, such descriptions of emergencies are normatively merged with the caller's first topic. As we have seen, this format seems to promote the progression of the interrogation, and partitions more clearly the responsibilities of the parties, explicitly assigning certain critical decisions to the professional party. Against the backdrop of considerable variation in call openings across emergency rescue agencies within as well as outside Europe, these findings allow us to argue for the potential benefits of designing the call-taking protocol as to allow for the integration of such preliminary institutional concerns within the native organisation of call openings. Such implementation strikes us as empirically well-founded, as well as relevant in relation to the institutional aims, policies, and practical work of emergency centres worldwide.

\section{References}

91/396/EEC: Council Decision of 29 July 1991 on the introduction of a single European 
emergency call number.

Arminen, I., Koskela, I. \& Vaajala, T. (2008). Configuring presence in simulated and mobile contexts. In: Anna Spagnolli \& Luciano Gamberini (Eds.), Proceedings of the $11^{\text {th }}$ Annual International Workshop on Presence. Padova, 16-18 October 2008.

Artman, H. \& Waern, Y. (1999). Distributed cognition in an emergency co-ordination centre. Cognition, Technology \& Work, 1, 237-246.

Baker, C. D., Emmison, M., \& Firth, A. (2005). Calling for Help: Language and Social Interaction in Telephone Helplines. John Benjamins: Amsterdam.

Bergmann, J. (1992). Alarmiertes Verstehen: Kommunikation in Feuerwehrnotrufen. In: Thomas Jung \& Stefan Müller-Doohm (Eds.), “Wirklichkeit” im Deutungsprocess. Frankfurt: Suhrkamp Verlag.

Bittner, E. (1974). The concept of organization. In: R. Turner (Ed.), Ethnomethodology. Harmondsworth: Penguin.

Cromdal, J. (in press.) Conversation analysis and emergency calls. In: Clair Chapelle (ed.), The Encyclopedia of Applied Linguistics. Oxford, UK: Wiley-Blackwell

Cromdal, J., Osvaldsson, K. \& Persson-Thunqvist, D. (2008). Context that matters: Producing "thick-enough descriptions" in initial emergency reports. Journal of Pragmatics, 40, 927-959.

Danby, S., Baker, C. D. and Emmison, M. (2005). Four observations on openings in calls to Kids Help Line. In: Carolyn D. Baker, Michael Emmison and Alan Firth, (Eds.), Calling for Help: Language and Social Interaction in Telephone Helplines. John Benjamins, Amsterdam. 133-151.

Drew, P. (1991). Asymmetries of knowledge in conversational interactions. In: I. Markovà, K. Foppa (eds.), Asymmetries in dialogue. Hemel Hempstead: Harvester Whetsheaf. 29-48

Edwards, D. (2007). Introduction. (Special issue titled "Calling for Help). Research on Language \& Social Interaction, 40, $1-7$.

Eglin, P. \& Wideman, D. (1979). Calling the police: Some aspects of the interactional organization of complaints in crime reporting. Analytic Sociology, 2.

Eglin, P. \& Wiedman, D. (1986). Inequality in professional service encounters: Verbal strategies of control versus task performance in calls to the police. Zeitschrift für Soziologie, 15, 341-362.

Emmison, M. \& Danby, S. (2007). Who's the friend in the background? Interactional strategies in determining authenticity in calls to a national children's helpline. Australian Review of Applied Linguistics, 30: 31.1-31.17.

Garcia, A. C. \& Parmer, P. A. (1999). Misplaced Mistrust: The Collaborative Construction of Doubt in 911 Emergency Calls. Symbolic Interaction, 22, 297-324.

Garfinkel, H. (1967). Studies in Ethnomethodology. Cambridge: Polity Press.

Heritage, J. \& Clayman, S. (2010). Talk in action: Interactions, identities and institutions. Chichester: Wiley-Blackwell.

Heritage, J. \& Raymond, G. (2005). The terms of agreement: Indexing epistemic authority and subordination in talk-in-interaction. Social Psychology Quarterly, 68,15-38.

Hopper, (1992). Telephone conversation. Bloomington: Indiana University Press.

Ikeya, N. (2003). Practical management of mobility: the case of the emergency medical system. Environment and Planning A, 35, 1547-1564.

Jefferson, G. (2004). Glossary of transcript symbols with an introduction. In: Gene H. Lerner, (Ed.), Conversation Analysis: Studies from the first generation. Amsterdam/Philadelphia: John Benjamins. 13-31.

Monzoni, C. (2009). Direct complaints in (Italian) calls to the ambulance: The use of negatively framed questions. Journal of Pragmatics, 41, 2465-2478.

Nordberg, B. (1998). On closings in alarm calls. Språk och stil, 8, 65-103. 
Normark, M. (2002). Using technology for real-time coordination of work. A study of work and artifacts in the everyday activities at SOS-Alarm. Stockholm: Royal Institute of Technology. (Diss).

Normark, M. \& Randall, D. (2005). Local expertise an an emergency call centre. In: H. Gellersen et al. (Eds.), ECSCW 2005: Proceedings of the Ninth European Conference on Computer-Supported Cooperative Work, 18-22 September 2005, Paris, France. 347-366.

Paoletti, I. (2007). Communication and gender issues in an Italian medical emergency control room: A case study. In: Mary Barrett \& Marilyn, J. Davidsson (Eds.), Gender and communication at work. Farnham: Gower. 166-179.

Parker, R., Pomerantz A, \& Fehr, B.J. (1995). Satisfaction Work in an Emergency Situation: The Case of the Philadelphia 911 Call. Journal of Consumer Satisfaction, Dissatisfaction, and Complaining Behaviour, 8, 164-176.

Pettersson, M. (2001). Talk and Visibility: 'One person is out of the car - Did you hear that?' Proceedings of the Iris 24. S. Björnestad, E. R. Moe, I. A. Mörch and A. L. Opdahl. 11th 14th of August, 2001, Ulvik, Hardanger, Norway, Iris. 24: 187 - 200.

Pettersson, M. (2004). WaterCalls: an ambient call queue for cooperation between emergency service centres. Personal and Ubiquitous Computing, 8, 192-199.

Pettersson, M., Randall, D. \& Helgeson, B. (2004). Ambiguities, awareness and economy: A study of emergency service work. Computer Supported Cooperative Work, 13, 125-154.

Sacks, H. (1972). On the analyzability of stories by children. In: J. J. Gumperz, and D. Hymes, (eds.), Directions in sociolinguistics: the ethnography of communication. New York: Rinehart \& Winston. 325-345

Sacks, H., Schegloff, E.A., \& Jefferson, G. (1974). A simplest systematics for the organization of turn-taking in conversation. Language, 50, 696-735.

Sacks, H. (1992). Lectures on conversation. Vols. 1-2., G. Jefferson (Ed.), London: Blackwells.

Schegloff, E. A. (1968). Sequencing in conversational openings. American Anthropologist, 70, 1075-1095.

Schegloff, E. A. (1979). Identification and recognition in telephone openings. In: G. Psathas (Ed.), Everyday language: Studies in ethnomethodology. N.Y.: Irvington Publishers. 2378.

Schegloff, E. A. (1986). The routine as achievement. Human Studies, 9, 111-52.

Schegloff, E. A. (2002). Reflections on research on telephone conversation: Issues of cross cultural scope and scholarly exchange, interactional import and consequences. In: Kang Kwong Luke, Theodossia-Soula Pavlidou (Eds.), Telephone Calls: Unity and diversity in conversational structure across languages and cultures. Amsterdam: John Benjamins. 249-81.

Schegloff, E. A. (2007). Sequence organisation in interaction: A primer in conversation analysis. Cambridge: Cambridge University Press.

Schegloff, E. A. \& Sacks, H. (1973). Opening up closings. Semiotica, 8, $289-327$.

Sharrock, W.W. \& Watson, D. (1989). Talk and police work: notes on the traffic in information. In: Hywel Coleman (Ed.), Working with language: A multidisciplinary consideration of language use in work contexts. Berlin: Mouton de Gruyter.

Turner, R. (1969). Occupational routines: Some demand characteristics of police work. Unpublished mimeographed paper presented to the Canadian Sociology and Anthropology Association. June 1969.

Wakin, M. A. \& Zimmmerman, D. H. (1999). Reduction and specialization in emergency and directory assistance calls. Research on Language and Social Interaction, 32, 409-437.

Watson, R. (1986). Doing the organization's work:An examination of aspects of the operation of a crisis intervention center. In: S. Fisher \& A. D. Todd, (Eds.), Discourse and 
institutional authority. Norwood, N.J.: Ablex.

Watson, R. \& Sharrock, W. (1990). Conversational actions and organisational actions. Reseaux, 8, 2, 21-34

Whalen, J., Zimmerman, D. H. \& Whalen, M. (1988). When words fail: A single case analysis. Social Problems, 35, 335-362.

Whalen, J. \& Zimmerman, D. H. (1998). Observations on the display and management of emotion in naturally occurring activities: The case of "hysteria" in calls to 9-1-1. Social Psychology Quarterly, 61, 141-159.

Whalen, J. \& Zimmerman, D. H. (2005). Working a call: Multiparty management and interactional infrastructure in calls for help. In: Carolyn D. Baker, Michael Emmison and Alan Firth, (Eds.), Calling for Help: Language and Social Interaction in Telephone Helplines. John Benjamins, Amsterdam. 309-345.

Whalen, M. R. \& Zimmerman, D. H. (1987). Sequential and institutional contexts in calls for help. Social Psychology Quarterly, 50, 172-185.

Whalen, M. R. \& Zimmerman, D. H. (1990). Describing trouble: Practical epistemology in citizen calls to the police. Language in Society, 19, 465-492.

Zimmerman, D. H. (1992a). Achieving context: openings in emergency calls. In: Graham Watson \& Robert Seiler (Eds.), Text in context: Contributions to ethnomethodology. London: Sage. 35-51.

Zimmerman, D. H. (1992b). The interactional organization of calls for emergency assistance. In: P. Drew \& J. Heritage, (Eds.), Talk at work. Cambridge: Cambridge University Press. 418-469.

\section{Appendix}

Transcription notations:

numbers in single parentheses represent pauses in seconds

micropause (shorter than .3s)

(( ))

analyst's comments

[

indicates start of simultaneous talk

]

indicates end of simultaneous talk

$=$

latched utterances

$(x)$

inaudible word

$(x x x)$

inaudible words

(drop dead)

best guess

$\rightarrow$

highlights a particular feature discussed in the text

prolongation of preceding sound

hi there

sounds marked by emphatic stress are underlined

HELLO

markedly increased amplitude 


\begin{tabular}{|c|c|}
\hline$\circ \circ$ & markedly lower amplitude \\
\hline$* *$ & hoarse voice \\
\hline$\sim \sim$ & wobbly voice \\
\hline$f €$ & smiling voice \\
\hline$\uparrow \downarrow$ & rising/falling intonation in succeeding syllable(s) \\
\hline ? & questioning intonation \\
\hline . & conclusion intonation \\
\hline- & abrupt halt \\
\hline$><$ & embeds talk that is faster than surrounding speech \\
\hline$<>$ & embeds talk that is slower than surrounding speech \\
\hline. $\mathrm{hh}$ & inbreath \\
\hline hh. & outbreath \\
\hline hi; ha; he; hö; hh & varieties of laughter \\
\hline ---01.30--- & call progression in minutes and seconds \\
\hline$\{\sim \sim \sim \sim \sim \sim\}$ & keyboard sound, timed with simultaneous talk \\
\hline
\end{tabular}

keynd, timed with simultaneous talk

\footnotetext{
The authors are greatly indebted to Bengt Nordberg for generously sharing the 90000 -corpus with us. An earlier version of this article was presented at a workshop titled "Mobilizing help", which was organised in June 2008 by Giolo Fele at University of Trento, as well as at a Talk-in-Interaction seminar (SIS) organised by Mathias Broth and the lead author at Linköping University. The participating colleagues - too many to list here - offered valuable reflections on an early version of the analysis. Many thanks to you all. Susan Danby and Mike Emmison generously commented on a final draft of this article. We are solely responsible for any remaining gaffs. This work was financially supported by a Bank of Sweden Research Grant (RJ, P2007-0837:1-E) awarded to the first author.

ii A comment on the translation is in place. Readers who lack linguistic access to the Swedish transcripts may be struck by the selection of the term "occurred", rather than its more mundane, and altogether more commonplace, equivalent "happened". The same, however, can be said about the Swedish term "inträffat", which readers familiar with spoken Swedish will identify as a very formal term, typically associated with language used in beaurocratic settings, written reports, and the like. According to our discussions with SOS Alarm officials, this question was indeed designed to highlight the formal, or official, status of the emergency dispatch agency - to provide a means for "doing authority" at the very outset of the call, so as to direct its future course in specific ways. Whether or not this goal is goal is achieved in actual practice is, of course, part of the subject matter of the analysis on hand.

iii For instance, in the case of the Australian Kids' HelpLine (KHL), Danby et al, (2005) show how call takers design their opening turn to allow for the caller to enter into the conversation in any way s/he may find suitable. Such a procedure constitutes one of many ways through which the overall policy of the help line "We care, we listen" - is interactionally produced, that is, oriented to and acted on, in the course of the call. iv This is provided for by the consistency rule - if one is mentioned (by the opening line) then the other is made relevant.
} 
Examples 3 and 4 need to be accounted for as well. The operator's question whether the burglary reported in example 3 is ongoing during the time for the call is clearly also about urgency (among other things). In example 4, the matter of urgency is already inferrable from the caller's report "kids running on the motorway". vi

See the Lane County 9-1-1 transcripts.

vii This should in no way imply that prior to 1996, callers were free to chose whatever emergency resources they saw fit. Clearly, all the dispatch-relevant decisions (including the type and scope of response actions) have always resided with the operator. This, of course, only points to the redundancy of callers requesting the dispatch of a particular type of response unit. Having said this, in some countries this choice is explicitly posed upon the caller. For instance, Ikeya (2003) reports from a corpus of 119-calls to a control-andcommand centre of a metropolitan fire department in Japan, showing emergency controllers responding to the summons with the following phrase: "Fire Department, fire or medical emrgency?".

viii Very briefly, such competence involves being familiar with relevant legal regulations (e.g., the requirement to notify police authorities, every time the fire brigade is alarmed), medical issues (e.g., pertaining to dispatcher's deciding on response priority) or general emergency logistics (e.g., current location of the nearest ambulance which, among other things, has implications for whether or not a more proximate fire brigade unit should be alerted to assist the victims in the meanwhile). It goes without saying that there are many more concerns involved in emergency call taking and dispatch work. Nonetheless, these are actual samples of the type of decisions which operators face in virtually every emergency situation. 